\title{
Adjuvante Therapieansätze in der Onkologie: Eine kritische Nutzen-Risiko-Abwägung
}

\author{
Ralf Gutzmer $^{\mathrm{a}^{*}} \quad$ Elke Jäger $^{\mathrm{b}^{*}} \quad$ Dirk Vordermark $\mathrm{c}^{\mathrm{c}^{*}}$ \\ ${ }^{a}$ Klinik für Dermatologie, Allergologie und Venerologie, Medizinische Hochschule Hannover, \\ ${ }^{\mathrm{b}}$ Medizinische Klinik Hämatologie - Onkologie, Krankenhaus Nordwest, Frankfurt/M., \\ ${ }^{c}$ Klinik für Strahlentherapie, Universitätsklinikum Halle/Saale, Deutschland
}

\section{Adjuvante Systemtherapie bei soliden Tumoren}

Konzepte zur adjuvanten Systemtherapie werden heute bei allen Patienten empfohlen, bei denen nach R0-Resektion ein hohes Rezidivrisiko besteht und aufgrund der Studienlage erwartet werden kann, dass durch eine postoperative Systemtherapie das progressionsfreie und möglicherweise auch das Gesamtüberleben verlängert wird. Bei der Therapieentscheidung im Einzelfall müssen neben den zu erwartenden Vorteilen allerdings auch potenzielle Beeinträchtigungen der Lebensqualität durch unerwünschte Ereignisse sowie die Zusatzkosten berücksichtigt werden.

Bei Patienten mit vollständig reseziertem Kolonkarzinom im Stadium III hat sich in den letzten Jahren die adjuvante Chemotherapie mit FOLFOX4 (Fluoruracil (5-FU)/Folinsäure + Oxaliplatin) als Standard etabliert. Grundlage hierfür waren die Ergebnisse der Phase-III-Studie MOSAIC, in der bei Patienten mit kurativ reseziertem Kolonkarzinom im Stadium II oder III das Oxaliplatin-haltige Schema mit dem früheren Standard 5-FU/Folinsäure verglichen wurde. Patienten im Stadium III hatten mit einer Verbesserung der krankheitsfreien 3-Jahres-Überlebensrate von $65,3 \%$ auf $72,2 \%$ einen statistisch signifikanten Vorteil von der Zugabe von Oxaliplatin; im Tumorstadium II war der Unterschied zwischen den beiden Therapiearmen dagegen nicht signifikant (84,3\% vs. $87,0 \%)$ [1].

Beim Rektumkarzinom wurde die früher übliche adjuvante Radiochemotherapie inzwischen durch Konzepte zur präoperativen Radiochemotherapie abgelöst, nachdem eine deutsche Arbeitsgruppe in einer randomisierten Studie für die präoperative Vorgehensweise eine statistisch signifikante Abnahme der Lokalrezidivrate nach 5 Jahren zeigen konnte ( $6 \%$ vs. $13 \%$; $p=0,006)$. Bei einer gleichzeitig signifikanten Abnahme der akuten und Langzeittoxizität waren die Unterschiede im krankheitsspezifischen und Gesamtüberleben allerdings nicht statistisch signifikant [2].

Konzepte zur adjuvanten und primär systemischen (neoadjuvanten) Therapie des Mammakarzinoms wurden unter der Vor-

*Alle Autoren haben zu gleichen Teilen zu diesem Artikel beigetragen. stellung entwickelt, dass es sich bei diesem Tumor um eine primär systemische Erkrankung handelt. Ziel der Systemtherapie ist es bei beiden Konzepten, über eine Reduktion der zirkulierenden Tumorzellen die Prognose zu verbessern. Die Äquivalenz der beiden Verfahren wurde in mehreren großen Studien gezeigt, wobei bei einer präoperativen Systemtherapie zugleich die Rate an brusterhaltenden Operationen auf über $90 \%$ stieg. Etwa $20 \%$ der Frauen erreichten eine pathologische Komplettremission, die als Surrogatmarker für ein längeres rezidivfreies und Gesamtüberleben gilt.

Den Einfluss des Patientenalters auf den Nutzen einer frühen Systemtherapie zeigten die Ergebnisse einer entsprechenden Analyse der Early Breast Cancer Trialists' Collaborative Group (EBCTCG). Von einer adjuvanten Chemotherapie profitierten danach vor allem jüngere Frauen unter 50 Jahren. In diesem Kollektiv war nach 15 Jahren die Rezidivrate $12,4 \%$ und die krankheitsspezifische Mortalität 10,0\% niedriger als im Kontrollarm. Bei älteren Frauen war der Vorteil mit 4,2\% bzw. 3,0\% dagegen relativ gering ausgeprägt [3].

Patientinnen mit HER2-positivem Mammakarzinom profitierten nach adjuvanter Chemotherapie \pm Strahlentherapie von der anschließenden Therapie mit Trastuzumab. In der 3-armigen HERA-Studie verbesserte die 1-jährige Therapie mit dem HER2-Antikörper das krankheitsfreie 2-Jahres-Überleben statistisch signifikant von 77,4\% auf 85,8\% (Hazard Ratio (HR) 0,54; 95\%-Konfidenzintervall (KI) 0,43-0,67; p < 0,0001) [4].

\section{Adjuvante Systemtherapie beim malignen Melanom}

Ausgehend von der Annahme, dass das maligne Melanom bei der großen Mehrzahl der Patienten nach der Markerhypothese metastasiert, d.h. die Tumorzellen simultan in regionäre Lymphknoten und entfernte Gewebe disseminieren, ist eine Verbesserung des fernmetastasenfreien und Gesamtüberlebens beim Melanom eher durch eine adjuvante Systemtherapie als durch lokoregionäre Interventionen zu erwarten.

\section{KARGER \\ Fax +497614520714 \\ Information@Karger.de}

www.karger.com 
Adjuvante Chemotherapie

Anders als beispielsweise beim Mammakarzinom konnte beim malignen Melanom für eine adjuvante Chemotherapie allerdings kein Überlebensvorteil gezeigt werden. In einer randomisierten Studie der German Dermatologic Cooperative Oncology Group, in der Hochrisiko-Patienten mit metastasiertem Melanom nach kompletter Metastasektomie mit Vindesin behandelt oder beobachtet wurden, war der Vorteil der adjuvant chemotherapeutisch behandelten Patienten weder beim krankheitsfreien Überleben ( $p$ $=0,40)$ noch bei der 3-Jahres-Gesamtüberlebensrate $(\mathrm{p}=0,07)$ statistisch signifikant [5]. Auch zahlreiche andere randomisierte Studien zeigten keine relevanten Vorteile einer adjuvanten Chemotherapie.

\section{Adjuvante Immuntherapien}

\section{Interferon alpha}

Als bisher einzige Substanz wurde Interferon alpha (rekombinantes INF alpha-2a und -2b) zur adjuvanten Therapie des malignen Melanoms zugelassen, nachdem mehrere randomisierte kontrollierte Studien sowohl für Hochdosis-Schemata als auch für Niedrigdosis-Schemata Vorteile im Vergleich zur alleinigen Beobachtung gezeigt hatten [6-8]. In einer 2010 veröffentlichten Metaanalyse, in die 8122 Patienten mit Hochrisiko-Melanom eingeschlossen wurden, verringerte Interferon alpha (verschiedene Dosierungsschemata) das relative Rezidivrisiko statistisch signifikant um 18\% (HR 0,82; $<<0,001)$ und das relative Mortalitätsrisiko um $11 \%$ (HR 0,89; $\mathrm{p}=0,002)$ [9].

Modifikationen der in den 1990er-Jahren entwickelten Hochdosis- und Niedrigdosis-Interferon-Therapieschemata zeigten bisher keine klinisch relevanten Vorteile. Ob die Prognose durch Einsatz von pegyliertem Interferon alpha verbessert werden kann, ist derzeit noch unklar. In der Phase-III-Studie EADO, in der bei Patienten mit einer Tumordicke $>1,5 \mathrm{~mm}$ und klinisch tumorfreien Lymphknoten Peginterferon alpha-2b (36 Monate) mit niedrig dosiertem Interferon alpha-2b (18 Monate) verglichen wurde, verbesserte das pegylierte Interferon weder das rezidivfreie Überleben noch das Gesamtüberleben [10]. Ergebnisse der von der Arbeitsgemeinschaft Dermatologische Onkologie (ADO) unter Leitung von Prof. Claus Garbe durchgeführten Studie zur Wirksamkeit von Peginterferon alpha-2a (vs. Niedrigdosis-INF alpha-2a) bei Patienten im Stadium IIA( $\left.\mathrm{T}_{3 \mathrm{a}}\right)$-IIIB liegen noch nicht vor.
Während die Frage nach dem im Einzelfall optimalen Therapieschema damit weiterhin offen ist, zeigten die Ergebnisse von zwei großen randomisierten Studien der European Organisation for Research and Treatment of Cancer (EORTC), dass von einer adjuvanten Therapie mit niedrig dosiertem Interferon (EORTC 18952) oder Peginterferon (EORTC 18991) besonders Patienten mit dicken Tumoren (Stadium IIB) oder Mikrometastasen (Stadium III N1) mit einer Verlängerung des fernmetastasenfreien und Gesamtüberlebens profitieren. Patienten mit Makrometastasen (Stadium III N2) hatten dagegen keinen Vorteil von den untersuchten Interferon-Schemata (Tab. 1). Eine weitere Subgruppenanalyse dieser EORTC-Studien konnte herausarbeiten, dass Patienten mit ulzeriertem Primärtumor besonders von einer adjuvanten Interferontherapie zu profitieren scheinen $[11,12]$.

\section{Vakzinierung mit MAGE-A3}

Beim malignen Melanom und verschiedenen anderen soliden Tumoren, wie nichtkleinzelligem Lungenkarzinom (NSCLC), Kopf-Hals-Tumoren und Harnblasentumor, wird das Melanoma-Antigen (MAGE)-A3-Protein auf Tumorzellen und Metastasen stabil exprimiert. Da MAGE-A3 auf gesunden Körperzellen nicht exprimiert wird, stellt das Protein eine vielversprechende Struktur für eine gezielte Immuntherapie dar. Nachdem MAGE-A3 in Kombination mit den Adjuvanzien AS028 oder AS15 bei Patienten mit MAGE-A3-positivem metastasierten Melanom hohe MAGE-A3-Antikörpertiter und T-Zellantworten induzierte [13], wird die Wirksamkeit der MAGE-A3-Vakzinierung in der adjuvanten Therapie zurzeit in einer großen internationalen Studie untersucht (Studienleitung in Deutschland: Medizinische Hochschule Hannover, Prof. Ralf Gutzmer). Primäres Ziel der doppelblinden, placebokontrollierten Phase-III-Studie (NCT00796445), in die weltweit 1300 Patienten mit MAGE-A3-positivem, reseziertem Melanom im Stadium III aufgenommen werden sollen, ist die Verbesserung des krankheitsfreien Überlebens.

\section{CTLA-4-Blockade}

Ebenfalls bei Hochrisiko-Patienten mit reseziertem Melanom im Stadium III wird derzeit in einer placebokontrollierten Phase-IIIStudie (NCT00636168) die Wirksamkeit eines CTLA-4 (cytotoxic T lymphocyte-associated antigen 4)-Antikörpers untersucht (Studienleitung in Deutschland: Universitätsklinikum Essen, Prof. Dirk Schadendorf). Primärer Studienendpunkt ist das rezidivfreie Überleben.
Tab. 1. EORTCStudien 18952 und 18991: Wirksamkeit von adjuvantem Interferon oder Peginterferon vs. Beobachtung in Abhängigkeit vom Tumorstadium (nach [11, 12])

\begin{tabular}{|c|c|c|c|c|c|}
\hline & \multicolumn{3}{|c|}{ EORTC 18952} & \multicolumn{2}{|c|}{ EORTC 18991} \\
\hline & \multicolumn{3}{|c|}{ Interferon alpha- $2 \mathrm{~b}$} & \multicolumn{2}{|c|}{ Peginterferon alpha- $2 \mathrm{~b}$} \\
\hline & \multicolumn{3}{|c|}{$\begin{array}{l}10 \text { Mio. I.E/Woche ( } 4 \text { Wochen) } \\
\rightarrow 5 \text { Mio. I.E./Woche ( } 24 \text { Monate) }\end{array}$} & \multicolumn{2}{|c|}{$\begin{array}{l}6 \mu \mathrm{g} / \mathrm{kg} / \text { Woche ( } 8 \text { Wochen) } \\
\rightarrow 3 \mu \mathrm{g} / \mathrm{kg} / \text { Woche ( } 58 \text { Monate) }\end{array}$} \\
\hline Stadium & $\mathrm{IIb}$ & III N1 & III N2 & III N1 & III N2 \\
\hline Patienten, $\mathrm{n}$ & 215 & 212 & 408 & 261 & 366 \\
\hline Fernmetastasenfreies Überleben, HR & 0,58 & 0,69 & 0,92 & 0,76 & 0,94 \\
\hline Gesamtüberleben, HR & 0,54 & 0,73 & 0,97 & 0,88 & 1,01 \\
\hline
\end{tabular}




\section{Adjuvante Therapieansätze in der Strahlentherapie}

Im Rahmen multimodaler Konzepte kann eine postoperative Radiotherapie bei verschiedenen soliden Tumoren die lokale bzw. lokoregionäre Tumorkontrolle verbessern. Eine positive Beeinflussung des krankheitsspezifischen und Gesamtüberlebens kann durch bereits vorliegende Fernmetastasen, initial bestehende Komorbiditäten sowie Toxizitäten der Strahlentherapie limitiert werden.

Als obligater Standard akzeptiert ist die adjuvante Strahlentherapie zum Beispiel bei der brusterhaltenden Therapie des Mammakarzinoms. Eindeutig empfohlen wird eine Radiotherapie in der aktuellen S3-Leitlinie bei Patientinnen mit Mastektomie und $>3$ befallenen axillären Lymphknoten $(\mathrm{pN}+)$ (Empfehlungsgrad 1); bei 1-3 befallenen Lymphknoten wird dagegen keine Empfehlung ausgesprochen (Empfehlungsgrad 0), obwohl verschiedene, allerdings ältere Studien auch in dieser Behandlungssituation einen Nutzen der adjuvanten Radiatio zeigten [14].

Beim NSCLC wurde die Indikation für eine postoperative Strahlentherapie aufgrund der in der PORT-Metaanalyse für die bestrahlten Patienten gesehenen Nachteile [15] in der Vergangenheit eher zurückhaltend gestellt. Nachdem neuere Daten aber in der Subgruppe der Patienten mit IIIA(N2)-Tumoren eine statistisch signifikante Verbesserung des krankheitsspezifischen und Gesamtüberlebens nach 5 Jahren zeigen konnten [16], sollte nach Angaben der aktuellen S3-Leitlinie bei Patienten im Stadium IIIA $_{1}$ und IIIA $_{2}$ die Indikation für eine postoperative Mediastinalbestrahlung geprüft werden [17].

\section{Adjuvante Strahlentherapie des malignen Melanoms}

Der therapeutische Stellenwert der adjuvanten Strahlentherapie beim malignen Melanom konnte aufgrund der bisher vorliegenden Daten noch nicht eindeutig definiert werden [18]. Retrospektive Analysen nicht randomisierter Studien bei Patienten mit Risikofaktoren für ein lymphogenes Lokalrezidiv (Kapseldurchbruch, Zweitrezidiv, extranodaler Befall, mehrere befallene Lymphknoten, Lymphknoten $>2$ bzw. $3 \mathrm{~cm}$ ), zeigten bei Hochrisiko-Patienten mit postoperativer Strahlentherapie relativ niedrige Lokalrezidivraten [19]. Die Ergebnisse der ersten randomisierten Studie, in der Patienten mit einem nach Lymphadenektomie geschätztem regionären Rezidivrisiko von $>25 \%$ bestrahlt (48 Gy in 20 Fraktionen) oder beobachtet wurden, zeigten nach einem medianen Follow-up von 27 Monaten eine statistisch signifikante Abnahme der Rezidivrate (20/109 vs. 34/108; HR 1,77; $\mathrm{p}=0,041)$. Eine statistisch signifikante Verbesserung des medianen Gesamtüberlebens konnte bisher allerdings nicht gezeigt werden (2,6 Jahre (RTx) vs. 3,9 Jahre (Beobachtung); $\mathrm{p}=0,14$ ) [20].

\section{Disclosure Statement}

Die Autoren waren Referenten beim «1. Interdisziplinäres Expertenforum Hautkrebs», das von Bristol-Myers Squibb unterstützt wurde.

\section{Literatur}

-1 André T, Boni C, Mounedji-Boudiaf L, et al.: Oxaliplatin, fluorouracil, and leucovorin as adjuvan treatment for colon cancer. N Engl J Med 2004; 350:2343-2351.

2 Sauer R, Becker H, Hohenberger W, et al.: Preoperative versus postoperative chemoradiotherapy for rectal cancer. N Engl J Med 2004;351:1731-1740.

$\checkmark 3$ Early Breast Cancer Trialists' Collaborative Group (EBCTCG): Effects of chemotherapy and hormonal therapy for early breast cancer on recurrence and 15-year survival: an overview of the randomised trials. Lancet 2005;365:1687-1717.

4 Piccart-Gebhart M, Procter M, Leyland-Jones B, et al.: Trastuzumab after adjuvant chemotherapy in HER2-positive breast cancer. N Engl J Med 2005; 353:1659-1672.

5 Eigentler TK, Radny P, Hauschild A, et al.: Adjuvant treatment with vindesine in comparison to observation alone in patients with metastasized melanoma after complete metastasectomy: a randomized multicenter trial of the German Dermatologic Cooperative Oncology Group. Melanoma Res 2008;18:353-358.

6 Kirkwood JM, Strawderman MH, Ernstoff MS, et al.: Interferon alfa-2b adjuvant therapy of high-risk resected cutaneous melanoma: the Eastern Cooperative Oncology Group Trial EST 1684. J Clin Oncol 1996;14:7-17.

7 Pehamberger H, Soyer HP, Steiner A, et al.: Adjuvant interferon alfa-2a treatment in resected primary stage II cutaneous melanoma. Austrian Malignant Melanoma Cooperative Group. J Clin Oncol 1998;16:1425-1429.
8 Grob JJ, Dreno B, de la Salmonière P, et al.: Randomised trial of interferon alpha-2a as adjuvant therapy in resected primary melanoma thicker than $1.5 \mathrm{~mm}$ without clinically detectable node metastases. French Cooperative Group on Melanoma. Lancet 1998;351:1905-1910.

-9 Mocellin S, Pasquali S, Rossi CR, et al.: Interferon alpha adjuvant therapy in patients with high-risk melanoma: a systematic review and meta-analysis. J Natl Cancer Inst 2010;102:493-501.

10 Grob JJ, Jouary T, Dreno B, et al.: Adjuvant therapy with pegylated interferon alfa- $2 \mathrm{~b}$ (36 months) versus low-dose interferon alfa-2b (18 months) in melanoma patients without macro-metastatic nodes: EADO trial. J Clin Oncol 2010;28(suppl 18s): abstr LBA8506.

11 Eggermont AM: Letter (Reply to F. Janku et al.). J Clin Oncol 2010;28:e17-e18.

12 Eggermont AM, Suciu S, Testori A, et al.: Ulceration of primary melanoma and responsiveness to adjuvant interferon therapy: Analysis of the adjuvant trials EORTC18952 and EORTC18991 in 2644 patients. J Clin Oncol 2009;27(suppl 15s):abstr 9007.

13 Kruit WH, Suciu S, Dreno B, et al.: Immunization with recombinant MAGE-A3 protein combined with adjuvant systems AS15 or AS02B in patients with unresectable and progressive metastatic cutaneous melanoma: A randomized open-label phase II study of the EORTC Melanoma Group (1603218031). J Clin Oncol 2008;26(suppl):abstr 9065.
14 Kreienberg R, Kopp I, Albert U, et al.: Interdisziplinäre S3-Leitlinie für die Diagnostik, Therapie und Nachsorge des Mammakarzinoms, Aufl. 2. München, Zuckschwerdt Verlag, 2008.

15 PORT Meta-Analysis Trialists Group: Postoperative radiotherapy in non-small-cell lung cancer: systematic review and meta-analysis of individual patient data from nine randomised controlled trials. Lancet 1998;352:257-263.

16 Lally BE, Zelterman D, Colasanto JM, et al.: Postoperative radiotherapy for stage II or III nonsmall-cell lung cancer using the surveillance, epidemiology, and end results database. J Clin Oncol 2006;24:2998-3006.

17 Goeckenjan G, Sitter H, Thomas M, et al.: Prävention, Diagnostik, Therapie und Nachsorge des Lungenkarzinoms. Pneumologie 2010;64(Suppl 2):S23S155.

18 Garbe C, Hauschild A, Volkenandt M, et al.: Evidence-based and interdisciplinary consensus-based German guidelines: systemic medical treatment of melanoma in the adjuvant and palliative setting. Melanoma Res 2008;18:152-160.

19 Guadagnolo BA, Zagars GK: Adjuvant radiation therapy for high-risk nodal metastases from cutaneous melanoma. Lancet Oncol 2009;10:409-416.

20 Henderson MA, Burmeister B, Thompson JF, et al.: Adjuvant radiotherapy and regional lymph node field control in melanoma patients after lymphadenectomy: Results of an intergroup randomized trial (ANZMTG 01,02/TROG 02,01). J Clin Oncol 2009;27(suppl 18s):abstr LBA9084. 